\title{
Outcomes Following Tele-rehabilitation in a Person with Parkinson Disease During the COVID-19
}

\section{Pandemic: A Case Report}

\section{Heather Barksdale, PT, DPT, NCS, MSCS}

UF Health Jacksonville, Outpatient Rehabilitation, Jacksonville FL, 4549 Emerson Street, Suite 300, Jacksonville FL 32207.

*Corresponding Author: Heather Barksdale, PT, DPT, NCS, MSCS, UF Health Jacksonville, Outpatient Rehabilitation, Jacksonville FL, 4549 Emerson Street, Suite 300, Jacksonville FL 32207.

Received date: 12 November 2021; Accepted date: 20 November 2021; Published date: 27 November 2021

Citation: Barksdale H (2021) Outcomes Following Tele-rehabilitation in a Person with Parkinson Disease During the COVID-19 Pandemic: A Case Report. J Med Case Rep Case Series 2(15): https://doi.org/10.38207/JMCRCS/2021/0215224

Copyright: (C) 2021 Heather Barksdale, PT, DPT, NCS, MSCS. This is an open-access article distributed under the terms of the Creative Commons Attribution License, which permits unrestricted use, distribution, and reproduction in any medium, provided the original author and source are credited.

Abstract
The COVID-19 pandemic has highlighted the need for alternative approaches to rehabilitation. While a few feasibility and pilot studies have
been published, there is currently little literature on assessment and intervention techniques for Physical Therapists (PTs) when utilizing
telerehabilitation (TR) for mobility impairments in individuals with underlying neurological deficits. This case report documents the adaptations
to care and functional outcomes of a TR plan of care for an individual with Parkinson's disease (PD) during the COVID-19 pandemic. The
participant was a 79-year-old African American woman, who was diagnosed with idiopathic PD. She demonstrated bradykinesia, hypokinesia,
impaired balance, trunk, and lower limb rigidity, resting tremor, and postural instability. The participant completed nineteen TR visits.
Improvements exceeding the minimal detectable change (MDC) values were noted on mobility assessments. The participant also reported high
satisfaction scores with the utilization of TR. PTs should consider the use of TR as an alternative to in-person rehabilitation for persons with
PD. Further research is required for guidelines on patient selection and generalizability.

\section{Introduction}

\section{Background and Purpose}

PD is a chronic and progressive neurodegenerative disorder of the basal ganglia. [1] Cardinal signs of the disease include bradykinesia, resting tremor, rigidity, and postural instability. [2] Symptoms can lead to deficits in standing balance, ambulation, functional transfers, and activities of daily living. Difficulty with these tasks can negatively affect the quality of life (QOL) for individuals with PD.

[3] In the United States, the prevalence of PD has been estimated to exceed 900,000 people with a prediction to rise to 1.2 million by 2030. [4]

Current medical management of PD includes surgical and pharmacological treatment. However, the importance of physical activity has also been a recent emphasis of comprehensive patient care for individuals with PD. [5] Research suggests that exercise is a low-risk, cost-effective intervention strategy that has a positive effect on both the motor and non-motor symptoms associated with PD. [6]

The National Parkinson Foundation found that individual's with PD who began an exercise regimen experienced a reduction in the rate of decline in QOL. [7]

While physical activity for individuals with PD has been identified as important, patient engagement in appropriate and effective interventions continues to be underutilized. [8] PTs are experts in the science of movement and prescribe individualized interventions to address specific symptoms experienced by individuals with PD. However, barriers continue to exist including transportation or proximity to neurological PT specialists. [9] The opportunity to utilize TR may alleviate these barriers to care. Telemedicine for individuals with PD has been shown to be a sustainable and efficient model of care. [10] However, few articles have been published on the use of TR for individuals with PD.

A recent systematic review assessed the use of telehealth for individuals with PD. [11] The review included twenty-one studies. Of these, a majority focused on remote monitoring or cognitive therapy. We are not aware of any studies that include the adaptations to TR sessions from usual care that can be replicated in a clinical capacity. This case report seeks to demonstrate the outcomes of TR and offer PTs insight into utilization for an individual with PD.

The COVID-19 pandemic has highlighted the need for alternative treatment platforms. While there is no current evidence that individuals with PD are at a higher risk of developing a severe form of COVID-19, individuals over fifty years of age and those with comorbidities have been. As the incidence of PD diagnosis increases with age, a majority of individuals with PD may fall into this higher risk category. [12] Recommendations have also been made for use of 
telemedicine for individuals with PD in order to enhance continuity of care during the pandemic [13] and calls for the development of

\section{Case Description}

Participant: The participant was a 79-year-old African American woman, diagnosed in 2012 with idiopathic PD, Hoehn \& Yahr stage 2. The participant received a referral to outpatient PT from her neurologist in December of 2020 during the COVID-19 pandemic. When the patient was contacted to schedule an evaluation, she refused services due to a history of co-morbidities including hypertension, hyperlipidemia, and heart disease with a pacemaker which placed her at a higher risk of severe illness and ICU admission. [15] This barrier to care was complicated by the participant's reliance on her caregiver for transportation needs as well as the caregiver's own fear of acquiring COVID-19. The participant was educated on the use of TR as a possible delivery method of PT services and advised of the risks and benefits of engaging in TR visits. Further explanation was also provided regarding the technical requirements to participate in TR including access to a high-speed internet connection and an available electronic device with both audio and video connections. Both the participant and her caregiver agreed to the need and use of TR as a medium for the delivery of care. An evaluation was then scheduled. The live TR sessions were performed utilizing a secured Zoom application built into the electronic medical record of the host facility The participant utilized an electronic tablet while the PT utilized a laptop for all of the TR sessions. Each had a built-in webcam. This case report format was reviewed by the University of Florida Institutional Review Board and found not to require submission. The participant did sign an authorization to use and disclose protected health information (PHI) in public activities, which was provided by the UF Health Jacksonville Privacy Office. All institutional policy

\section{Tests and Measures}

All outcome measures were assessed at the initial examination to establish baseline performance and immediately following completion of the intervention visits. Functional outcomes were also completed at the tenth visit per Medicare guidelines. The same PT assessed outcome measures and performed intervention treatment sessions. The PT was board certified in neurology and had over twelve years of experience treating patients with neurological deficits in the outpatient setting.

The participant performed a series of movements that were demonstrated by the PT and then mirrored by the participant. Movement testing assessed quality and degree of motion including identification of underlying postural and coordination impairments. The participant was observed demonstrating symptoms of bradykinesia, hypokinesia, impaired sitting and standing balance, trunk and lower limb rigidity, reduced lower extremity strength and telehealth programs specifically including rehab services have also been made. [14]

Health Insurance Portability and Accountability Act requirements for disclosure of protected health information were followed.

\section{Evaluation}

At initial evaluation, the PT confirmed aspects of the participant's environment including verification of those able to hear PHI, explanation of procedure in case of an emergency, and confirmation of the participant's physical location including address. The participant also affirmed that she was able to see and hear the PT. These aspects of care were established based on safety, billing, and APTA practice plan recommendations. The caregiver provided a "tour" of the environment where the therapy sessions would take place. Recommendations were made to remove tripping hazards and reduce external distractions. Instructions on setup for safe performance of the therapeutic exercise program and improvement in a viewing of the session were also provided. The participant requested that the caregiver be present for all sessions. The PT agreed and instructed the caregiver on assistance with guarding and movement of technology as needed.

The participant completed a verbal history intake including details of past medical history, home environment, the current level of participation, and patient goals. Prior surgical history included a lumbar fusion (L4-L5) performed over two years prior with no remaining precautions or residual pain. The participant also reported that she was compliant with her PD medication (Carbidopa-Levodopa $25 / 100$, four times a day) and denied notable on and off times or symptoms of freezing. The participant's goal for PT was to improve the safety and duration of standing and walking in order to help more around the house.

range of motion, limited trunk rotation, and upper extremity resting tremor. Gait impairments were observed including a bilateral decrease in step length (shuffling), decreased heel contact bilaterally, widened base of support, difficulty with turning, festination, and decreased gait speed, as well as postural instability. These impairments led to an increased risk of falls and limited household and community ambulation.

A functional assessment was performed with the participant using a rolling walker while the caregiver guarded at a stand-by assist level and included the 5XSTS ${ }^{(43-46)}$ and the BBS ${ }^{(47-49)}$. The participant also completed self-assessment surveys to include the PDQ-39(50) and FOTO ${ }^{(51)}$. Following completion of the intervention visits, the participant completed a patient satisfaction survey regarding the use of TR. 


\section{Intervention}

The participant completed eighteen treatment visits, one time a week for thirty-minute sessions. caregiver assisted in facilitating guarding during balance assessment and testing, holding and changing the position of the tablet for improved session viewing, and facilitating the home program in between PT guided sessions. Home exercise sheets were emailed utilizing an encrypt and send feature to the participant after each session.

Therapeutic exercises were evidence-based and selected to directly address the participant's observed impairments and functional deficits. These interventions included a component of amplitudespecific training, [16] high-intensity resistance training, [17] and trunk rotation interventions. [18] A summary of the interventions and timeline of progressions are listed in Table 1. Interventions were performed with PT demonstration. This approach allowed the participant to mirror the PT's movements accurately with the required amplitude, direction, and level of exertion.

Table 1. Intervention Summary

\begin{tabular}{|c|c|c|c|c|}
\hline & Visits 1-4 & Visits 5-9 & Weeks 10-13 & Weeks 14-19 \\
\hline \multicolumn{5}{|l|}{ Amplitude specific training } \\
\hline Stepping forward & $1 \times 10^{\mathrm{b}}$ & $2 \times 10^{b}$ & $2 \times 10^{c}$ & $2 \times 10^{c}$ \\
\hline Stepping backward & $1 \times 10^{\mathrm{b}}$ & $2 \times 10^{\mathrm{b}}$ & $2 \times 10^{c}$ & $2 \times 10^{c}$ \\
\hline Stepping sideways & $1 \times 10^{\mathrm{b}}$ & $2 \times 10^{\mathrm{b}}$ & $2 \times 10^{c}$ & $2 \times 10^{c}$ \\
\hline Sit to stand & $1 \times 10^{\mathrm{b}}$ & $1 \times 10^{\mathrm{b}}$ & $1 \times 10^{\mathrm{d}}$ & $1 \times 10^{\mathrm{d}}$ \\
\hline \multicolumn{5}{|c|}{ Weight Bearing/Resistance Training } \\
\hline Squats & & $1 \times 10^{\mathrm{b}}$ & $1 \times 10^{c}$ & $1 \times 10^{c}$ \\
\hline Calf raises & & $1 \times 10^{\mathrm{b}}$ & $1 \times 10^{c}$ & $1 \times 10^{c}$ \\
\hline Lunges & & $1 \times 10^{\mathrm{b}}$ & $1 \times 10^{c}$ & $1 \times 10^{c}$ \\
\hline \multicolumn{5}{|l|}{ Trunk Rotation Tasks } \\
\hline Across body reach & $1 \times 10^{a}$ & $1 \times 10^{c}$ & $1 \times 10^{c}$ & $1 \times 10^{\mathrm{d}}$ \\
\hline Overhead reach with rotation & $1 \times 10^{\mathrm{a}}$ & & $1 \times 10^{c}$ & $1 \times 10^{\mathrm{d}}$ \\
\hline Step turns- 90 degrees & & & $1 \times 10^{\mathrm{b}}$ & $1 \times 10^{c}$ \\
\hline \multicolumn{5}{|l|}{ aseated. } \\
\hline \multicolumn{5}{|l|}{${ }^{\mathrm{b}}$ Two hand hold on walker. } \\
\hline \multicolumn{5}{|l|}{ 'One hand hold on walker. } \\
\hline dNo hand hold. & & & & \\
\hline
\end{tabular}

\section{Outcomes}

Improvements on the BBS, 5XSTS and the mobility portion of the PDQ-39 exceeded MDC values following completion of the intervention visits. The 5XSTS score improved by more than 18 seconds and progressed from two to no handhold. The BBS score improved by sixteen points indicating a reduction in fall risk. Scoring of individual items of the BBS and PDQ-39 is provided in Tables 2 and 3. Any changes on the subsets besides mobility of the PDQ-39 did not meet their respective MDC values. The participant also self- reported improvement in the quality of her walking, duration of stance prior to requiring a rest break, and ability to stand to fold laundry as compared to before engaging in the TR sessions.

The participant reported high satisfaction related to the use of TR. A summary of the follow-up TR-specific patient satisfaction questionnaire is provided in Table 4. Additional patient satisfaction scores and specific patient-reported functional questions as determined by FOTO are presented in Table $\mathbf{5}$. 
Table 2. Berg Balance Scale

\begin{tabular}{llll}
\hline Item & Initial Evaluation & Visit 10 & After Intervention \\
\hline Sitting to Standing & & 4 & 4 \\
Standing Unsupported & 3 & 4 & 4 \\
Sitting Unsupported & 3 & 4 & 4 \\
Standing to Sitting & 4 & 4 & 4 \\
Transfers & 3 & 4 & 4 \\
Standing with eyes closed & 3 & 3 & 3 \\
Standing with feet together & 0 & 3 & 3 \\
Reaching forward with an outstretched arm & 3 & 3 & 0 \\
Retrieving an object from the floor & 2 & 0 & 4 \\
Turning to look behind & 0 & 3 & 3 \\
Turning 360 degrees & 3 & 2 & 3 \\
Placing alternate foot on stool & 2 & 2 & 2 \\
Standing with one foot in front of the other & 2 & 0 & 0 \\
Standing on one foot & 0 & 0 & $44 / 56^{\mathrm{a}}$ \\
Total (out of 56 points) & 0 & $36 / 56^{\mathrm{a}}$ & \\
\hline
\end{tabular}

${ }^{\mathrm{a}}$ Exceeds minimal detectable change value of 5 points.

Table 3. Parkinson's Disease Questionnaire

\begin{tabular}{lll}
\hline Category Subset & Initial Evaluation & After Intervention \\
\hline Mobility & 36 & $29^{\mathrm{a}}$ \\
Activities of Daily Living & 11 & 11 \\
Emotion & 12 & 9 \\
Stigma & 0 & 0 \\
Social & 0 & 1 \\
Cognition & 10 & 11 \\
Communication & 4 & 4 \\
Bodily Pain and Discomfort & 5 & 6 \\
\hline
\end{tabular}

${ }^{a}$ Exceeds minimal detectable change value as established for that subset

Table 4. Tele-Rehabilitation Specific Patient Satisfaction Questionnaire

\begin{tabular}{|c|c|}
\hline Subjective Question & Response After Intervention \\
\hline I feel that my provider understood me while using tele-rehabilitation & Strongly Agree $^{\mathrm{a}}$ \\
\hline I could understand my provider while using tele-rehabilitation & Strongly Agree ${ }^{a}$ \\
\hline I feel that the quality of care that I received was good & Strongly Agree ${ }^{a}$ \\
\hline I did not wait too long to see my provider & Strongly Agree ${ }^{a}$ \\
\hline This visit did not take too much time & Strongly Agree ${ }^{\mathrm{a}}$ \\
\hline If I had to travel to see my provider, it would be easy for me to do so & Strongly Disagree ${ }^{a}$ \\
\hline How satisfied were you with the overall use of tele-rehabilitation? & Extremely Satisfied ${ }^{\mathrm{b}}$ \\
\hline How satisfied were you with the types of exercises provided through tele-rehabilitation? & Extremely Satisfied $^{\mathrm{b}}$ \\
\hline Have you participated in outpatient rehabilitation (physical therapy) before? & Yes \\
\hline What issues or barriers of care did you have with outpatient rehab? & Transportation \\
\hline $\begin{array}{l}\text { Did the tele-rehabilitation program help alleviate any of these previously mentioned issues } \\
\text { or barriers? }\end{array}$ & Yes, absolutely. \\
\hline
\end{tabular}

What improvements (if any) did you notice physically after utilizing tele-rehabilitation? Walking better, standing longer, can fold laundry standing now. 
Were there any problems encountered while using tele-rehabilitation?

Anything else that you would like us to know about the tele-rehabilitation program?
No

The whole experience was a good one overall.

aScale included the following answer options: "Strongly Agree, Agree, Neutral, Disagree, Strongly Disagree".

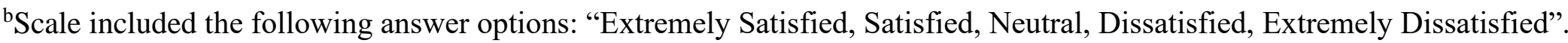

Table 5. Focus on Therapeutic Outcomes Self-Reported Satisfaction and Outcomes

\begin{tabular}{|c|c|c|}
\hline Subjective Question & $\begin{array}{l}\text { Initial } \\
\text { Evaluation }\end{array}$ & $\begin{array}{l}\text { After } \\
\text { Intervention }\end{array}$ \\
\hline How limited would you be walking one block? & Limited a lot ${ }^{\mathrm{a}}$ & Limited a little $^{a}$ \\
\hline How limited would you be walking around a room? & Not limited at all ${ }^{\mathrm{a}}$ & Not limited at all ${ }^{\mathrm{a}}$ \\
\hline How limited would you be climbing one flight of stairs? & Limited a lot ${ }^{\mathrm{a}}$ & Limited a lot ${ }^{\mathrm{a}}$ \\
\hline How limited would you be bathing or dressing? & Limited a little $^{a}$ & Limited a little $^{a}$ \\
\hline How limited would you be getting in and out of a chair? & Limited a little $^{\mathrm{a}}$ & Limited a little $^{\mathrm{a}}$ \\
\hline $\begin{array}{l}\text { How satisfied were you with the information about your } \\
\text { condition? }\end{array}$ & N/A & Very Satisfied $^{b}$ \\
\hline $\begin{array}{l}\text { How satisfied were you with the input in setting your treatment } \\
\text { goals? }\end{array}$ & N/A & Very Satisfied $^{\mathrm{b}}$ \\
\hline $\begin{array}{l}\text { How satisfied were you with the level of courtesy and respect } \\
\text { shown by the treatment team? }\end{array}$ & N/A & Very Satisfied $^{\mathrm{b}}$ \\
\hline $\begin{array}{l}\text { How satisfied were you with the availability of convenient } \\
\text { appointments? }\end{array}$ & N/A & Very Satisfied $^{b}$ \\
\hline How satisfied were you with the treatments for your condition? & N/A & Very Satisfied $^{b}$ \\
\hline How satisfied were you with the overall results of treatment? & $\mathrm{N} / \mathrm{A}$ & Very Satisfied $^{\mathrm{b}}$ \\
\hline \multicolumn{3}{|c|}{ "Scale included the following answer options: "Limited a lot, limited a little, Not limited at all" } \\
\hline
\end{tabular}

\section{Discussion}

The participant in this study demonstrated improvement with all functional measures as well as self-reported QOL measures. Improvements on the BBS and 5XSTS reflected a decrease in fall risk and correlated with the participant's self-reported improvements in function. These improvements are consistent with previous studies examining the effect of outpatient rehabilitation interventions on static balance, fall risk, and functional lower limb muscle strength. [19]

While the mobility subset of the PDQ-39 improved to meet the MDC, other subsets did not. This was not unexpected, as the intervention was not focused on those areas of care and points to the importance of task specificity in rehabilitation. However, the subsets of emotion, cognition, and bodily pain and discomfort were rated as slightly improved when compared to before the intervention. Further investigation may be indicated to address the lack of evidence on the effect of TR on these aspects of quality of life.
PTs often express concerns regarding the use of TR with their patients.[20] These concerns include safety, use of technology, and lack of equipment in the home. This participant did not experience any falls or other adverse events while participating in the TR sessions. All visits were completed as scheduled with no dropped Zoom calls. The participant did not have any exercise equipment available at home, but the session was adapted to incorporate bodyweight and items easily available to the patient in order to facilitate the program instead. Future research should continue to explore trends in these aspects of care in order to establish guidelines for PTs.

A larger study is needed to establish the efficacy of the use of TR for individuals with PD. A study is currently in the development phase at UF Health Jacksonville and will incorporate information gained through the findings of this case report in its design. 


\section{Limitations}

This case report examined one participant's experience and performance with TR. While the participant demonstrated improvement in mobility scores, reported positive changes related to the quality of life, and reported high satisfaction with the use of TR, this was a single case. Further research is required for information on patient selection and generalizability. Longitudinal studies should be conducted to establish the amount of TR sessions needed to make

\section{Summary}

The results of his case report suggest that TR may be a safe and effective mode of delivery for individuals with idiopathic PD. PTs may want to consider the use of TR as an alternative to in-person rehabilitation for persons with $\mathrm{PD}$.

\section{Declarations}

The author does not have any conflicts of interests or funding sources to disclose.

\section{References}

1. Obeso J, Marin C, Rodriguez-Oroz C, Blesa J, Benetiz-Temino B, et al. (2008) The Basal Ganglia in Parkinson's disease: Current concepts and unexplained observations. Ann Neurol. 64(Suppl 2): S30-S46.

2. Postuma R, Berg D, Stern M, Poewe W, Olanow CW, et al. (2015) MDS clinical diagnostic criteria for parkinson's disease. Movement Disorders. 30(12): 1591-1601.

3. Rahman S, Griffin H, Quinn N, Jahanshahi M (2008) Quality of life in Parkinson's disease: The relative importance of they symptoms. Movement Disorders. 23(10): 1428-34.

4. Foundation. Ps. Prevalence Project. 2021.

5. Lauze M, Daneault J-F, Duval C (2016) The effects of physical activity in Parkinson's disease: A review. Journal of Parkinson's Disease. 6(4): 685-698.

6. Bhalsing K, Abbas M, Tan L (2018) Role of physical activity in Parkinson's disease. Ann Indian Acad Neurol. 21(4): 242-249.

7. Rafferty M, Schmidt P, Luo S, Li K, Marras C, et al. (2017) Regular exercise, quality of life, and mobility in Parkinson's disease: A longitudinal analysis of National Parkinson Foundation quality improvement initiative data. J Parksinsons Dis. 7(1): 193-202.

8. Hunter H, Lovegrove C, Haas B, Freeman J, Gunn H (2019) Experiences of people with Parkinson's disease and their views on physical activity interventions: a qualitative systematic review. JBI Database of Systematic Reviews and Implementation Reports. 17(4): 548-613.

9. Lageman S, Mickens M, Cash T (2015) Caregiver-identified needs and barriers to care in Parkinson's disease. Geriatric Nursing. 36(3): 197-201. functional improvements as well as to determine how long these changes in mobility remain after completion of the therapy.

Another limitation identified was a lack of observational measures to identify aspects of the quality of ambulation. While an observational gait assessment was performed, a Functional Gait Assessment or Timed Up and Go may have provided additional information on the participant's improvement with dynamic balance and quality of ambulation.

The work has not been presented to previous scientific meetings or publications.

Acknowledgments: The author wishes to thank Abby Jones, SPT for her assistance with review of pertinent outcome measures and Sarah Mersch OTR/L, CHT for her assistance with proofreading.

10. Schneider R, Biglan K (2017) The promise of telemedicine for chronic neurological disorders: the example of Parkison's disease. Lancet Neurol. 16(7): 541-551.

11. Chen Y-Y, Guan B-S, Li Z-K, Yang Q-H, Xu T-J, et al. (2020) Application of telehealth intervention in Parkinson's disease: A systematic review and meta-analysis. Journal of Telemedicine and Telecare. 26(1-2): 3-13.

12. Papa SM, Brundin P, Fung VSC, Kang UJ, Burn DJ, et al. (2020) Telemedicine in neurological disorders: Opportunities and challenges. Telemedicine and E-Health. 25(7): 541-550.

13. Miele G, Straccia G, Moccia M, Leocani L, Tedeschi G, et al. (2020) Telemedicine in Parkinson's disease: How to ensure patient needs and continuity of care at the time of COVID-19 pandemic. Telemedicine and E-Health. 26(12): 1533-1536.

14. Feeney MP, Xu Y, Surface M, Shah H, Vanegas-Arroyave N, et al. (2021) The impact of COVID-19 and social distancing on people with Parkinson's disease: a survey study. Parkinson's Disease. 7(1): 10

15. Jian V, Yuan JM (2020) Predictive symptoms and comorbidities for severe COVID-19 and intensive care unit admissions: a systematic review and meta-analysis. International Journal of Public Health. 25 : 1-14.

16. Farley BG, Fox CM, Ramig L, McFarland D (2008) Intensive Amplitude-specific therapeutic approaches for Parkinson's disease: Toward a neuroplasticity-principled rehabilitation model. Topics in Geriatric Rehabilitation. 24(2): 99-114.

17. Dibble L, Hale TF, Marcus R, Gerber P, LaStoya P (2009) High intensity eccentric resistance training decreased bradykinesia and improves quality of life in persons with Parkinson's disease: A 
preliminary study. Parkinsonism \& Related Disorders. 15(10): 752-7.

18. Bartolo M, Serrao M, Tassorelli C, Don R, Ranavolo A, et al. (2010) Four-week trunk-specific rehabilitation treatment improves lateral trunk flexion in Parkinson's disease. Movement Disorders. 25(3): 325-31.

19.
Chow B, Feloiu F, Berardocco A, Ceglie D, Nesathurai S (2020)

Functional Improvement Related to Enrolment in a Parkinson's

Disease Rehabilitation Program. Neurorehabilitation. 47(4): 405414.

20. Shaw DK (2009) Overview of telehealth and its application to cardiopulmonary physical therapy. Cardiopulmonary Physical Therapy Journal. 20(2): 13-8. 